\title{
Novel Treatment for Radiation Optic Neuropathy With Intravenous Bevacizumab
}

\author{
Osman Farooq, MD, Norah S. Lincoff, MD, Nicolas Saikali, MD, \\ Dheerendra Prasad, MD, MCh (Neurosurgery), Robert S. Miletich, MD, PhD, \\ Laszlo L. Mechtler, MD
}

\begin{abstract}
Radiation optic neuropathy is a devastating form of vision loss that can occur months to years after radiation therapy for tumors and other lesions located in close proximity to the visual pathways. We present the case of a 24-year-old woman who underwent external beam radiation for treatment of a tectal pilocytic astrocytoma, and 5 years later she developed bilateral radiation optic neuropathy and radiation necrosis of the right temporal lobe. We opted to treat her with intravenous bevacizumab with 3 doses every 3 weeks, as well as dexamethasone and pentoxifylline. After the first infusion of bevacizumab, the patient noted improvement in vision and color vision, and a follow-up magnetic resonance imaging study showed that the previous enhancement of the optic nerves and chiasm was diminishing. Her vision improved dramatically and has remained stable over a 3-year period.
\end{abstract}

Journal of Neuro-Ophthalmology 2012;32:321-324

doi: $10.1097 /$ WNO.0b013e3182607381

() 2012 by North American Neuro-Ophthalmology Society

$\mathbf{R}$ adiation optic neuropathy (RON) typically leads to devastating vision loss in one or both eyes (1-3). Recently, there have been reports of anti-vascular endothelial growth factors used in the treatment of radiation damage, both in the eye (4-7) and the central nervous system (CNS) (8-11). We report the use of this therapeutic agent in a patient with RON.

School of Medicine (OF, NSL, NS, DP, RSM, LLM), Departments of Neurology (OF, NSL, NS, LLM), Ophthalmology (NSL), Radiation Medicine (DP), and Nuclear Medicine (RSM), University of Buffalo, Buffalo, New York; Jacobs Neurologic Institute (OF, NSL), Buffalo, New York; Women and Children's Hospital of Buffalo (OF), Buffalo, New York; Ross Eye Institute (NSL), Buffalo, New York; Dent Neurologic Institute (NS, RSM, LLM), Buffalo, New York; and Roswell Park Cancer Institute (DP, LLM), Buffalo, New York.

The authors have no funding or conflicts of interest to declare. Address correspondence to: Norah S. Lincoff, MD, Jacobs Neurologic Institute, Buffalo General Hospital, 100 High Street, Buffalo, NY 14203; E-mail: lincoff@buffalo.edu

\section{CASE REPORT}

A 26-year-old woman presented 10 years previously with headaches and somnolence. At that time, brain magnetic resonance imaging (MRI) revealed a tectal mass that was thought to be a pilocytic astrocytoma. Eight years ago, she developed obstructive hydrocephalus as a result of the growth of the tumor (Fig. 1). The patient underwent left ventriculostomy and resection of the tumor that confirmed the diagnosis. She received intensity-modulated radiation therapy of $54 \mathrm{~Gy}$ in 30 fractions at $1.8 \mathrm{~Gy}$ per fraction. Mean doses were as follows: right optic nerve $4.4 \mathrm{~Gy}$ (range: 1.2-10.3 Gy), left optic nerve 6.6 Gy (range: 2.8-10.4 Gy), and optic chiasm 24.8 Gy (range: 11.4-45.8 Gy). A Varian 21/CD linear accelerator using $6 \mathrm{MeV}$ photons, with a 56 leaf (1-cm leaf width), multileaf collimator and sliding window technique was used (Fig. 2). Three months later, the patient underwent a second craniotomy for tumor

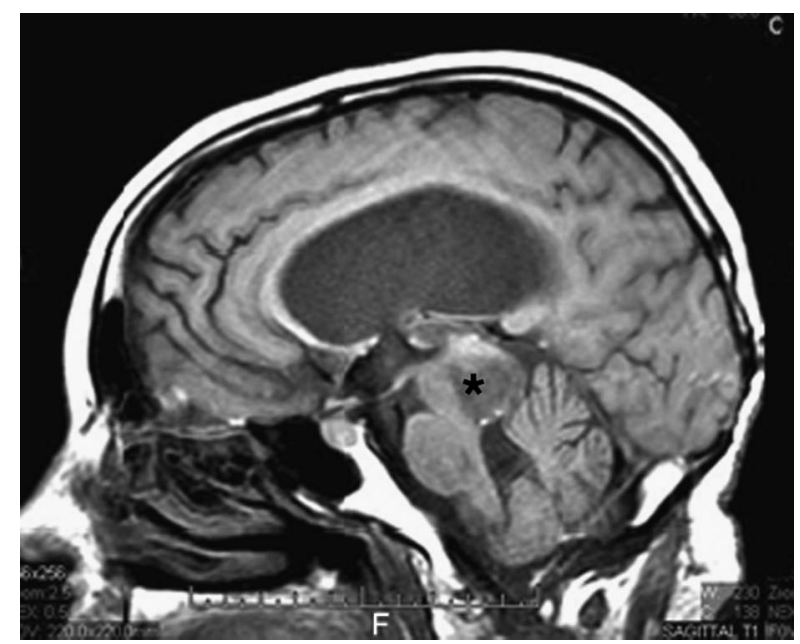

FIG. 1. T1 sagittal magnetic resonance imaging (MRI) shows tectal tumor (asterisk). 


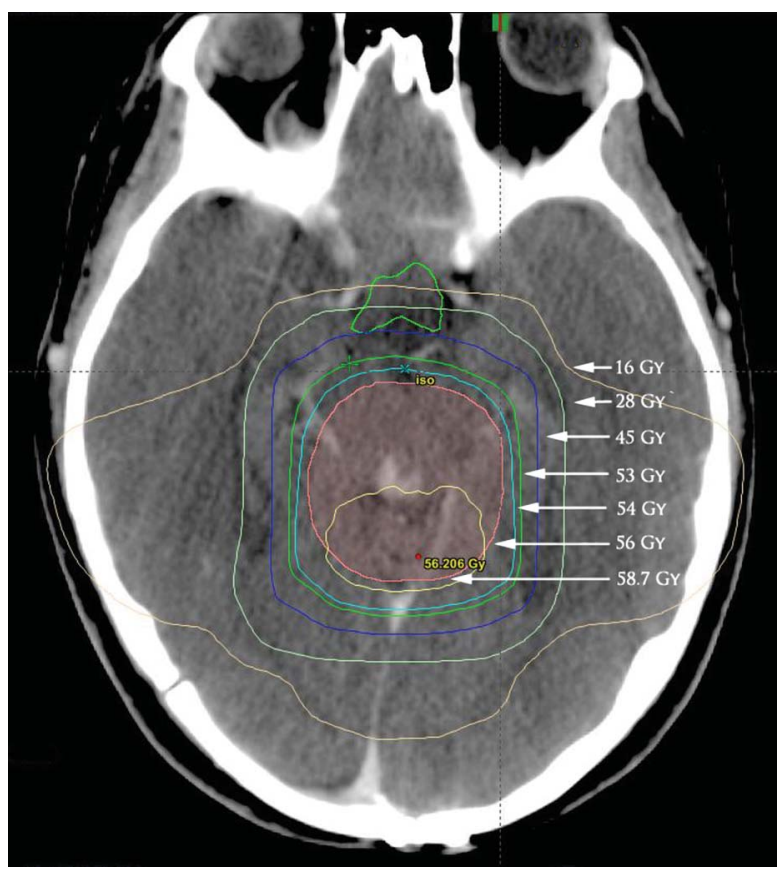

FIG. 2. T1 axial magnetic resonance imaging (MRI) with distribution of radiation isodose curves (Gy).

recurrence, followed by treatment with carboplatin/vincristine chemotherapy for 15 months.

Five years and 3 months later, the patient reported headaches and vision loss in the right eye. She described her vision as "white." Visual acuity was 20/80, right eye, and 20/40, left eye. Brain MRI showed a subcortical area of hyperintensity in the right temporal lobe (Fig. 3). This was thought to be consistent with radiation damage, and the

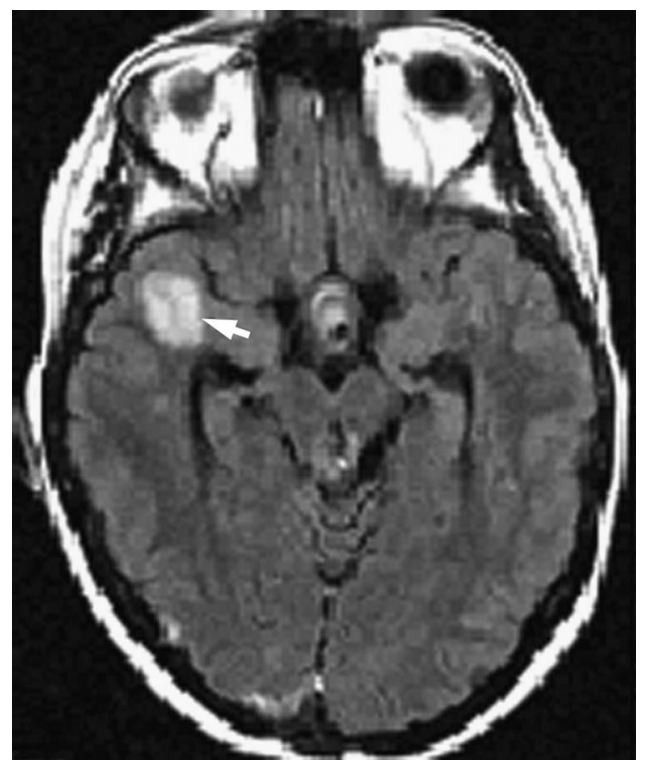

FIG. 3. Contrasted T1 axial magnetic resonance imaging (MRI) with subcortical hyperintensity in the right temporal lobe (arrow).

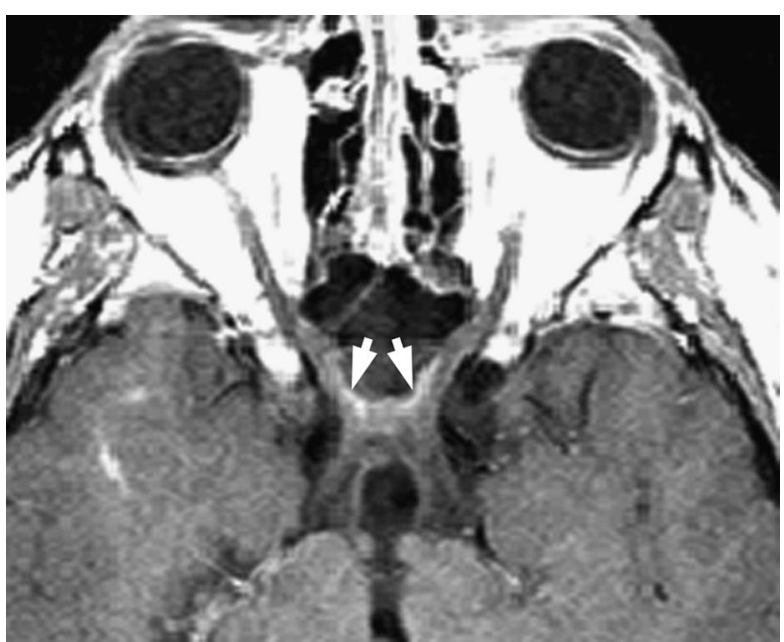

FIG. 4. Contrast-enhanced $T 1$ axial image shows areas of enhancement along both optic nerves (arrows).

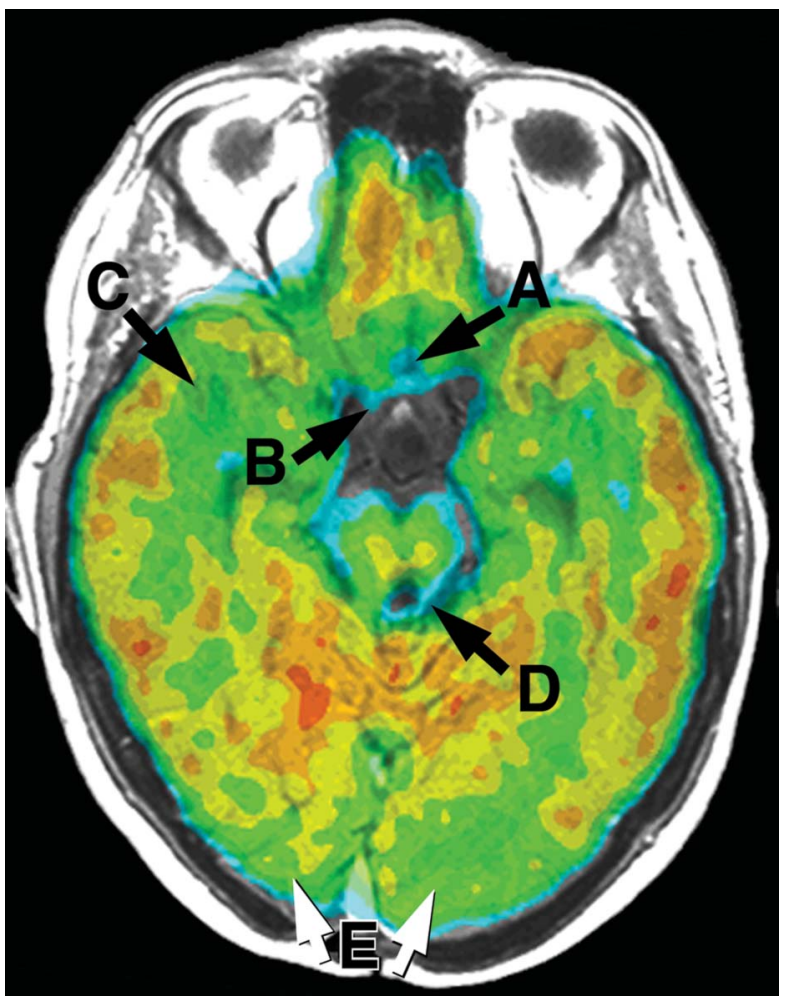

FIG. 5. Positron emission tomography (PET) from PET/computed tomography registered to contrast-enhanced magnetic resonance imaging (MRI) before bevacizumab therapy. Automated image registration demonstrates stereotactic concordance of MRI and PET changes. MRI is rendered in gray scale and fluorodeoxyglucose (FDG)-PET in color spectrum (metabolism: high to minimal, red to blue to gray). There is FDG uptake in the chiasm (A) and the proximal component of the right optic tract (B). The right temporal lobe lesion has peripheral contrast-enhancement and shows FDG uptake slightly greater than normal white matter $(\mathbf{C})$. There is a surgically created cavity at the tectum with adjacent metabolically active tissue (D). Diffuse occipital cortical hypometabolism (white arrows) (E) is present in a pattern consistent with diminished afferent input from the anterior visual pathways. 

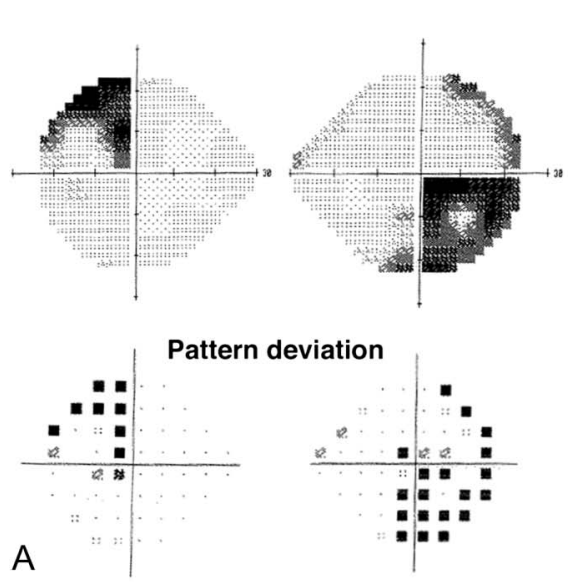
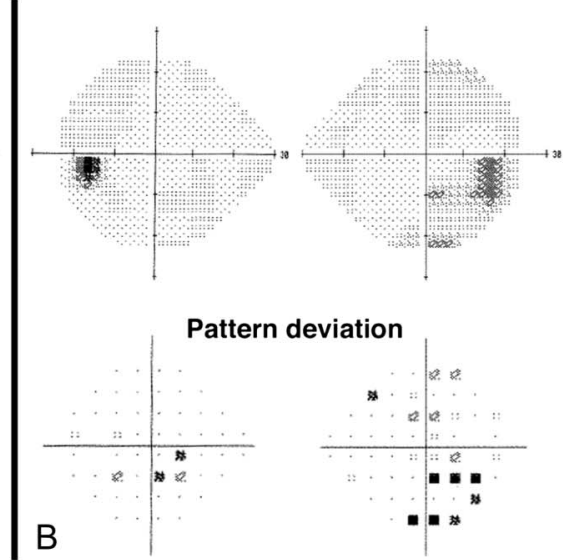

FIG. 6. A. Automated visual fields demonstrate an incomplete bitemporal hemianopia. B. After treatment, there is marked improvement in the visual field defects.

patient was started on intravenous methylprednisolone for 5 days, followed by $4 \mathrm{mg}$ of dexamethasone daily.

Two weeks later, optic nerve enhancement was noted bilaterally (Fig. 4), which also was felt to be caused by radiation damage. The dose of dexamethasone was increased to $4 \mathrm{mg}$ twice daily. Visual acuity was no light perception, right eye, and 20/100, left eye. Positron emission tomography (PET) was consistent with inflammatory changes of radiation injury (Fig. 5).

Four weeks after the onset of visual loss, the patient was given $675 \mathrm{mg}$ of intravenous bevacizumab $(7.5 \mathrm{mg} / \mathrm{kg})$ with 3 doses every 3 weeks, as well as $8 \mathrm{mg}$ of dexamethasone per day and $400 \mathrm{mg}$ of pentoxifylline per day. MRI scanning 2 weeks after initiating treatment with bevacizumab showed decreasing enhancement of the optic nerves and chiasm.

During the second week of treatment, the patient noted improvement in her visual acuity and reported periods of extreme clarity for 5 to 10 minutes at a time. Visual acuity was 20/50, right eye, and 20/100, left eye. The patient identified the color plates slowly, but correctly, in each eye. Pupils reacted poorly to light, and automated visual fields revealed a bitemporal hemianopia (Fig. 6A). Bilateral optic atrophy was present. Over the next 4 weeks, her subjective episodes of clarity increased to hours at a time, and she regained acuity of 20/25, right eye, and 20/40, left eye, with further improvement in her visual fields (Fig. 6B).

Six weeks after initiation of bevacizumab, brain MRI revealed complete resolution of the areas of enhancement (Fig. 7). With 3 years of follow-up, the patient's vision has stabilized at 20/20, right eye, and 20/25, left eye. Color vision is intact bilaterally. Subtle bitemporal visual field defects remain, and both optic discs are pale.

\section{DISCUSSION}

This report suggests that intravenous bevacizumab may be an effective treatment of RON. Our patient noted dramatic improvement in her vision within 4 weeks of initiation of therapy, and there was complete resolution of brain and optic nerve enhancement within 6 weeks. Over a 3-year period, her visual function has remained stable.

Bevacizumab has been reported to be effective in the treatment of radiation necrosis of the CNS. Gonzalez et al (8) demonstrated the benefit of using bevacizumab or
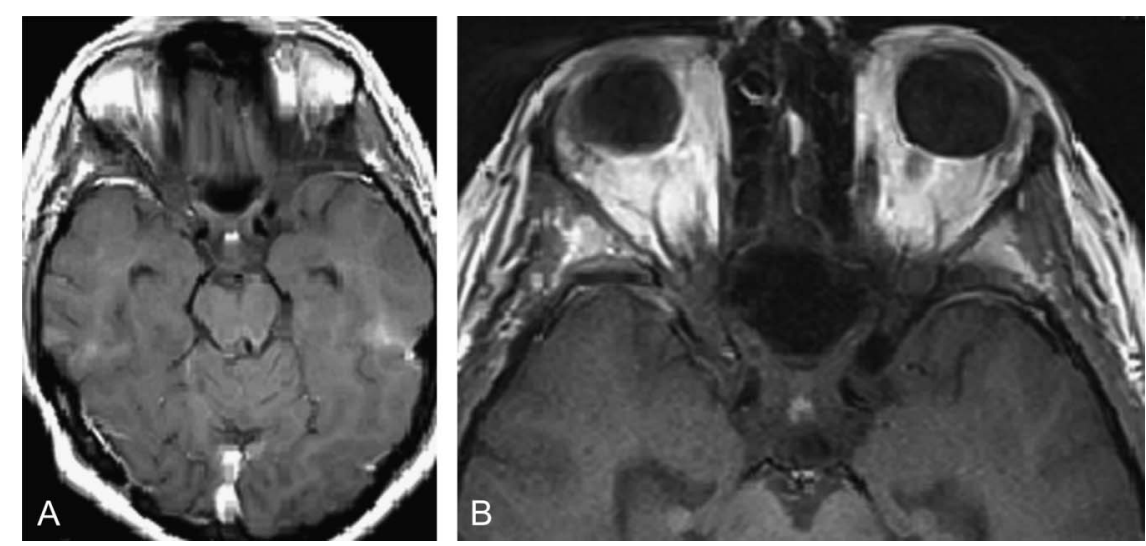

FIG. 7. Six weeks after beginning bevacizumab treatment, the areas of enhancement in the right temporal lobe (A) and optic nerves $(\mathbf{B})$ are no longer present. 
bevacizumab with a chemotherapeutic agent (carboplatin, irinotecan, temozolomide) in 15 patients diagnosed with cerebral radiation necrosis. All patients showed improvement in both fluid-attenuated inversion recovery (FLAIR) and postcontrast T1 MRI abnormalities at an average of eight weeks after beginning bevacizumab. Torcuator et al (10) reported 6 patients with biopsy-proven cerebral radiation necrosis treated with bevacizumab between 2006 and 2008 and documented improvement in both FLAIR and contrast-enhanced T1 images. Levin et al (12) postulated that aberrant production of vascular endothelial growth factor is involved with radiation necrosis of the brain and that even short treatment (fewer than four doses) with bevacizumab seems to turn off the cycle of radiation damage. Levin et al (12) enrolled 14 patients into a placebocontrolled, randomized, double-blind study to evaluate the effect of bevacizumab in treating CNS radiation necrosis. In evaluating MRI findings, 0 of 7 patients receiving placebo responded, whereas all 5 of 5 randomized and 7 of 7 crossover patients showed regression of necrotic lesions. Only 2 patients had recurrence of MRI findings, and both were retreated with bevacizumab.

Adverse effects from systemic administration of bevacizumab include cardiovascular (hypertension, thromboembolism), CNS (headache, pain syndromes, tumor recurrence), gastrointestinal (abdominal pain, nausea, vomiting, anorexia), hematologic (hemorrhage, leukopenia, neutropenia), and musculoskeletal (weakness, myalgias) abnormalities (13-15). These adverse affects are fortunately rare, and none were observed in our patient.

Although pentoxifylline was part of our patient's treatment protocol, its therapeutic efficacy is unproven. This hemorrheologic methylxanthine derivative might increase tissue perfusion by reducing platelet aggregation and reduce cytokine-mediated inflammation by inhibiting tumor necrosis factor and fibroblast growth factor 2. Yet in a comprehensive review, Nieder et al (16) found "little evidence" that pentoxifylline has any significant effect on radiation therapy. We thus believe that it was the bevacizumab that was responsible for the patient's visual improvement.

We acknowledge that our findings will need to be confirmed in a randomized trial to determine the optimal duration and effectiveness of treatment in a large study group. However, in the interim, we recommend that bevacizumab be considered in patients suffering acute visual loss from RON.

\section{REFERENCES}

1. Levy RL, Miller NR. Hyperbaric oxygen therapy for radiationinduced optic neuropathy. Ann Acad Med Singapore. 2006;35:151-157.

2. Mihalcea 0, Arnold AC. Side effect of head and neck radiotherapy: optic neuropathy. Oftalmologia. 2008;52:36-40.

3. Arnold AC. Radiation optic neuropathy. Presented at: North American NeuroOphthalmology Society Meeting; February 1923, 1995; Tucson, AZ.

4. Atkins EJ, Bruce BB, Newman NJ, Biousse V. Treatment of nonarteritic anterior ischemic optic neuropathy. Surv Ophthalmol. 2010;55:47-63.

5. Finger PT, Chin K. Anti-VEGF bevacizumab (Avastin) for radiation optic neuropathy. Am J Ophthalmology. 2007;143:335-338.

6. Hosseini H, Razeghinejad MR. Anterior ischemic optic neuropathy after intravitreal injection of bevacizumab. J Neuroophthalmol. 2009;29:160-161.

7. Finger $\mathbf{P}$, Chin K. Anti-vascular endothelial growth factor bevacizumab (Avastin) for radiation retinopathy. Arch Ophthalmol. 2007;125:751-756.

8. Gonzalez J, Kumar A, Conrad C, Levin V. Effect of bevacizumab on radiation necrosis of the brain. Int J Radiat Oncol. 2007;67:323-326.

9. Sheline GE, Wara WM, Smith V. Therapeutic irradiation and brain injury. Int J Radiat Oncol. 1980;6:1215-1228.

10. Torcuator R, Zuniga R, Mohan YS, Rock J, Doyle T, Anderson J, Gutierrez J. Ryu S, Jain R, Rosenblum M, Mikkelsen T. Initial experience with bevacizumab treatment for biopsy confirmed cerebral radiation necrosis. J Neurooncol. 2009;94:63-68.

11. LeBas J. Treating radiation necrosis on the brain. OncoLog. 2009;54.

12. Levin VA, Bidaut L, Hou P, Kumar AJ, Wefel JS, Bekele BN, Prabhu S, Loghin M, Gilbert MR, Jackson EF. Randomized double-blind placebo-controlled trial of bevacizumab therapy for radiation necrosis of the central nervous system. Int J Radiat Oncol Biol Phys. 2011;79:1487-1495.

13. Ranpura V, Hapani S, Wu S. Treatment-related mortality with bevacizumab in cancer patients. JAMA. 2011;305:487-494.

14. Bukowski RM. Metastatic clear cell carcinoma of the kidney: therapeutic role of bevacizumab. Cancer Manag Res. 2010;2:83-96.

15. Johnson DH, Fehrenbacher L, Novotny WF, Herbst RS, Nemunaitis JJ, Jablons DM, Langer CJ, DeVore RF, Gaudreault J, Damico LA, Holmgren E, Kabbinavar F. Randomized Phase II Trial comparing bevacizumab plus carboplatin and paclitaxel with carboplatin and paclitaxel alone in previously untreated locally advanced or metastatic nonsmall-cell lung cancer. J Clin Oncol. 2004;22:2184-2191.

16. Nieder C, Zimmermann FB, Adam M, Molls M. The role of pentoxifylline as a modifier of radiation therapy. Cancer Treat Rev. 2005;31:448-455. 